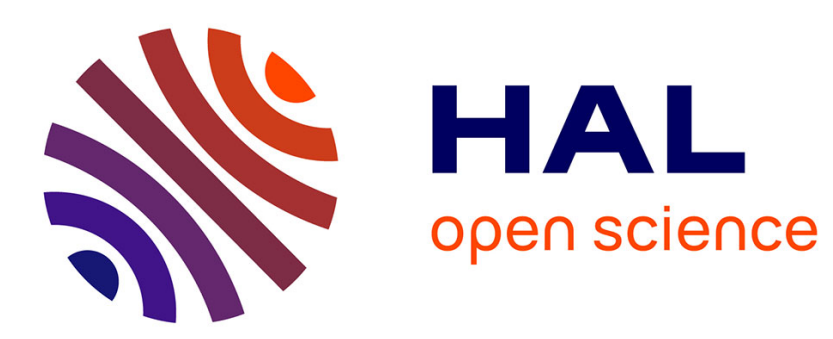

\title{
Statistics on Lie groups: a need to go beyond the pseudo-Riemannian framework
}

\author{
Nina Miolane, Xavier Pennec
}

\section{To cite this version:}

Nina Miolane, Xavier Pennec. Statistics on Lie groups: a need to go beyond the pseudo-Riemannian framework. Bayesian inference and Maximum Entropy Methods in Science and Engineering (MaxEnt 2014), Sep 2014, Amboise, France. pp.59-66, 10.1063/1.4905963 . hal-01091515

\section{HAL Id: hal-01091515 https://inria.hal.science/hal-01091515}

Submitted on 5 Dec 2014

HAL is a multi-disciplinary open access archive for the deposit and dissemination of scientific research documents, whether they are published or not. The documents may come from teaching and research institutions in France or abroad, or from public or private research centers.
L'archive ouverte pluridisciplinaire HAL, est destinée au dépôt et à la diffusion de documents scientifiques de niveau recherche, publiés ou non, émanant des établissements d'enseignement et de recherche français ou étrangers, des laboratoires publics ou privés. 


\title{
Statistics on Lie groups : a need to go beyond the pseudo-Riemannian framework
}

\author{
Nina Miolane and Xavier Pennec \\ INRIA, Asclepios project-team, \\ 2004 Route des Lucioles, BP93, F-06902 Sophia Antipolis Cedex, France
}

\begin{abstract}
Lie groups appear in many fields from Medical Imaging to Robotics. In Medical Imaging and particularly in Computational Anatomy, an organ's shape is often modeled as the deformation of a reference shape, in other words: as an element of a Lie group. In this framework, if one wants to model the variability of the human anatomy, e.g. in order to help diagnosis of diseases, one needs to perform statistics on Lie groups. A Lie group $\mathscr{G}$ is a manifold that carries an additional group structure. Statistics on Riemannian manifolds have been well studied with the pioneer work of Fréchet, Karcher and Kendall [1, 2, 3, 4] followed by others [5, 6, 7, 8, 9]. In order to use such a Riemannian structure for statistics on Lie groups, one needs to define a Riemannian metric that is compatible with the group structure, i.e a bi-invariant metric. However, it is well known that general Lie groups which cannot be decomposed into the direct product of compact and abelian groups do not admit a bi-invariant metric. One may wonder if removing the positivity of the metric, thus asking only for a bi-invariant pseudo-Riemannian metric, would be sufficient for most of the groups used in Computational Anatomy. In this paper, we provide an algorithmic procedure that constructs bi-invariant pseudo-metrics on a given Lie group $\mathscr{G}$. The procedure relies on a classification theorem of Medina and Revoy. However in doing so, we prove that most Lie groups do not admit any biinvariant (pseudo-) metric. We conclude that the (pseudo-) Riemannian setting is not the richest setting if one wants to perform statistics on Lie groups. One may have to rely on another framework, such as affine connection space.
\end{abstract}

Keywords: Lie group, Lie algebra, Statistics, pseudo-Riemannian PACS: $02.20 . \mathrm{Sv}, 02.40 . \mathrm{Ky}, 02.40 . \mathrm{Re}$

\section{INTRODUCTION}

D'Arcy Thompson proposes in 1917 to model any anatomy as a diffeomorphic deformation of a reference shape [10], hence as an element of an infinite dimensional Lie group. In this context, if one wants to perform statistics on anatomies, one needs a statistical framework on Lie groups. In order to train our intuition, we consider finite dimensional Lie groups here. To define a statistical framework, it seems natural to start with the definition of a mean. On a Lie group, this definition should be consistent with the group structure. This means that a left or right translation of the data set should translate its mean accordingly. Also, the inversion of all data elements should lead to an inverted mean. A mean verifying these properties is said bi-invariant. A bi-invariant candidate for the mean on Lie groups is the group exponential barycenter defined in [11] through the canonical Cartan-Shouten connection. We call it the bi-invariant mean. Its local existence and uniqueness has been proven if the dispersion of the data is small enough, more precisely if the data are in a sufficiently small normal convex neighborhood of some point $g$ of the Lie group. In this paper, we investigate geometric structures on 
Lie groups that could help to provide a computational framework for the bi-invariant mean. In particular, we are interested in characterizing global domains of existence and uniqueness of the bi-invariant mean, for example a ball of maximal radius such that any probability measure with support included in it would have a unique bi-invariant mean.

A Lie group is a manifold that carries an additional group structure. Several definitions of the mean on Riemannian manifolds have been proposed in the literature: the Fréchet mean the Karcher mean or the Riemannian exponential barycenter [1, 2, 3, 4, 5, 6]. The Riemannian framework provides theorems for their global existence and uniqueness domains $[5,6,7,8,9]$, ensuring the computability of statistics on Riemannian manifolds. One may wonder if we could use this framework for statistics on Lie groups, and more particularly for the bi-invariant mean. In fact, a Riemannian mean coincides with the group exponential barycenter if and only if the Riemannian metric is itself biinvariant. In this case, the Riemannian geodesics coincide with the geodesics of the Cartan-Schouten connection [12]. However, a Lie group does not have any bi-invariant Riemannian metric in general. The real Lie groups $S T(n)$, the Heisenberg group $H$, $U T(n)$ and $S E(n)$ don't have any bi-invariant metric while they admit a locally unique bi-invariant mean [11]. Thus, the Riemannian framework is not the good setting to characterize the bi-invariant mean. The pseudo-Riemannian framework is a tempting alternative. The class of Lie groups that admit a bi-invariant pseudo-metric is larger than the class of those with a bi-invariant metric. We could try to generalize the Riemannian statistical framework to a pseudo-Riemannian statistical framework and apply it for Lie groups. For instance, the mean on a pseudo-Riemannian manifold could be defined as a critical point of the variance (defined with the pseudo-distance induced by the pseudometric). Of course, uniqueness theorems would have to be established. But the first issue is: how many Lie groups do admit a bi-invariant pseudo-metric? Is it the case for the real Lie groups $S T(n), H, U T(n)$ and $S E(n)$, which have a locally unique bi-invariant mean?

Our contribution is an algorithmic reformulation of a classification theorem for Lie algebras $[13,14]$ that answers theses questions. Taking a Lie group $\mathscr{G}$ as input, the algorithm constructs a bi-invariant pseudo-metric on $\mathscr{G}$ in case of existence. Using this algorithm, we show that most Lie groups that have a locally unique bi-invariant mean do not possess a bi-invariant pseudo-metric. We conclude that, for the purpose of statistics on real Lie groups -and more precisely for the computational framework of the biinvariant mean- it is not worth generalizing the Riemannian statistical framework to a pseudo-Riemannian framework. The paper is organized as follow. In the first section, we introduce notions of Lie algebra. In the second section, we present the (tree-structured) algorithm. In the third section, we apply the algorithm on $S T(n), H, U T(n)$ and $S E(n)$ to show that most of them do not have a bi-invariant pseudo-metric.

\section{NOTIONS OF LIE ALGEBRA}

In this section, we present the algebraic notions underlying the construction of the algorithm. We consider finite dimensional connected Lie groups over $\mathbb{F}=\mathbb{R}$ or $\mathbb{C}$.

Quadratic Lie groups. A Lie Group $\mathscr{G}$ is a smooth manifold with a compatible group structure. Its tangent space at the identity $\left(\mathfrak{g}=T_{e} \mathscr{G},+, .,[,]_{\mathfrak{g}}\right)$ is called its Lie algebra. 
We can define a pseudo-metric $<,>$ on $\mathscr{G}$, making it a pseudo-Riemannian manifold. A left-invariant pseudo-metric is a pseudo-metric $<,>$ s.t. $\left\langle D L_{h}(g) X, D L_{h}(g) Y\right\rangle$ $\left.\right|_{L_{h} g}=\left\langle X, Y>\left.\right|_{g}\right.$ for all $X, Y \in T_{g} \mathscr{G}$ and all $g, h \in \mathscr{G}$, where $L_{h}$ is the left translation by $h$. Similarly, we can define right-invariant and bi-invariant $<,>$. Lie groups that admit a bi-invariant $\langle$,$\rangle are called quadratic Lie groups. A Lie group is quadratic$ if and only if its Lie algebra $\mathfrak{g}$ has a non-degenerate symmetric quadratic form (non necessarily positive), also denoted $<,>$, verifying [12]: $\forall x, y, t \in \mathfrak{g},<[x, y]_{\mathfrak{g}}, t>+<$ $y,[x, t]_{\mathfrak{g}}>=0$ (1). Because of the finite dimension, we rewrite Eq.(1) as a set of $\operatorname{dim}(\mathfrak{g})$ matrix equations: $\forall x \in \mathscr{B}_{\mathfrak{g}}, \quad M(x)^{T} . Z+Z \cdot M(x)=0$ (2) where $\mathscr{B}_{\mathfrak{g}}$ is a basis of $\mathfrak{g}, M(x)$ the matrix of the endomorphism $a d(x): y \mapsto[x, y]$, and $Z$ a symmetric invertible (non necessarily positive) matrix representing $<,>$ on $\mathscr{B}_{\mathfrak{g}}$. Computing bi-invariant $<,>$ on $\mathscr{G}$ amounts to solve Eq.(1) for $<,>$ or Eq.(2) for $Z$. The (symmetric invertible) solutions span a vector space, the quadratic space $\mathscr{Z}(\mathfrak{g})$, of dimension $d_{q}(\mathfrak{g})$, the quadratic dimension of $\mathfrak{g}$.

Lie algebra representations. A $\mathfrak{g}$-representation on the vector space $V$ is a Lie algebra homomorphism $\eta: \mathfrak{g} \mapsto \mathfrak{g l}(V)$. A $\mathfrak{g}$-subrepresentation is a subspace of $V$ stable by the elements of $\eta(\mathfrak{g})$. An irreducible $\mathfrak{g}$-subrepresentation is a $\mathfrak{g}$-subrepresentation without proper $\mathfrak{g}$-subrepresentation. An indecomposable $\mathfrak{g}$-subrepresentation is a $\mathfrak{g}$ subrepresentation that can not be decomposed into $\mathfrak{g}$-subrepresentations. Note that irreducibility implies indecomposability but the converse is false : it is not always possible to decompose a $\mathfrak{g}$-representation into irreducible $\mathfrak{g}$-subrepresentations, only into indecomposable ones. Two g-representations $\eta_{1}$ on $V_{1}$ and $\eta_{2}$ on $V_{2}$ are isomorphic if there is an isomorphism of representations between them, i.e. an isomorphism of vector spaces $l: V_{1} \mapsto V_{2}$ s. t.: $\eta_{2}(x) \circ l=l \circ \eta_{1}(x)$. We write $V_{1} \sim V_{2}$. Taking $V=\mathfrak{g}$, we define the adjoint representation of $\mathfrak{g}$, ad $: \mathfrak{g} \mapsto \mathfrak{g l}(\mathfrak{g})$ by $x \mapsto \operatorname{ad}(x)=[x, \bullet]_{\mathfrak{g}}$. For ad, $\mathfrak{g}$-subrepresentations are ideals of $\mathfrak{g}$, irreducible $\mathfrak{g}$-representations are minimal ideals of $\mathfrak{g}$, indecomposable $\mathfrak{g}$-representations are ideals of $\mathfrak{g}$ that can not be decomposed into a direct sum of ideals of $\mathfrak{g}$. If ad is irreducible, $\mathfrak{g}$ is said to be simple. On the dual $\mathfrak{g}^{*}$, we define the co-adjoint representation $\theta: x \mapsto \theta(x)=-<\bullet, a d(x)>$ where $\langle$,$\rangle is the$ canonical product defining the dual basis. Note that solving Eq.(2) amounts to find a symmetric isomorphism of representations $Z$ between ad and $\theta$.

Direct sum. Take $\left(B,[,]_{B}\right)$ a Lie algebra. $B=B_{1} \oplus B_{2}$ is the direct sum of $B_{1}, B_{2}$ if:

- $B=B_{1} \oplus B_{2}$ in terms of vector spaces,

- $\left[B, B_{1}\right]_{B} \subset B_{1}$, and $\left[B, B_{2}\right]_{B} \subset B_{2}$, making $B_{1}$ and $B_{2}$ ideals of $B$.

This definition is equivalent to the decomposition of the adjoint representation of $B$ into the $B$-representations $B_{1}$ and $B_{2}$ i.e.: $\operatorname{ad}(b)=[b, \bullet]_{B}=\left(\begin{array}{cc}\operatorname{ad}\left(b_{1}\right) & 0 \\ 0 & \operatorname{ad}\left(b_{2}\right)\end{array}\right)$ on a basis respecting $B_{1} \oplus B_{2}$. Now, $B$ being quadratic is equivalent to $B_{1}$ and $B_{2}$ being quadratic. If $<,>_{B_{1}},<,>_{B_{2}}$ are bi-invariant on $B_{1}, B_{2}$ and represented by the matrices $Z_{B_{1}}, Z_{B_{2}}$ then:

$$
Z_{B_{1} \oplus B_{2}}=\left(\begin{array}{cc}
Z_{B_{1}} & 0 \\
0 & Z_{B_{2}}
\end{array}\right)
$$


is bi-invariant on $B$. Conversely, if $<,>_{B}$ is bi-invariant on $B$, its restrictions $<,>\left._{B}\right|_{B_{1}}$ and $<,>\left._{B}\right|_{B_{2}}$, are bi-invariant on $B_{1}, B_{2}$. In practice, computing the direct sum decomposition of $B$ into indecomposable $B_{i}$ 's amounts to a simultaneous bloc diagonalization of the adjoint representatives of elements of $\mathscr{B}_{B}$, a basis of $B$.

Double extension. $\quad B=W \oplus S \oplus S^{*}$ is the double extension of $W$ by a simple $S$ if:

- $B=W \oplus S \oplus S^{*}$ in terms of vector spaces,

- $\left(W,[,]_{W}\right)$ is a Lie algebra and $[S, W]_{B} \subset W$ makes $W$ a $S$-representation,

- $\left(S,[,]_{S}\right)$ is a simple Lie subalgebra of $B:\left[s, s^{\prime}\right]_{B}=\left[s, s^{\prime}\right]_{S}$,

- $S^{*}$ is the dual space of $S$ and $\left[S, S^{*}\right]_{B} \subset S^{*}$ makes $S^{*}$ the co-adjoint representation,

- $\forall w, w^{\prime} \in W: \quad\left[w, w^{\prime}\right]_{B}=\left[w, w^{\prime}\right]_{W}+\beta\left(w, w^{\prime}\right)$ where $\beta: \Lambda^{2} W \mapsto S^{*}$ is an $S$ equivariant map, ie a map that commutes with the action of $S$.

This definition is equivalent to the following decomposition of the adjoint representation of $B: \operatorname{ad}(b)=[b, \bullet]_{B}=\left(\begin{array}{ccc}{[w, \bullet]_{W}+[s, \bullet]_{B}} & {[w, \bullet]_{B}} & 0 \\ 0 & {[s, \bullet]_{S}} & 0 \\ \omega(w, \bullet) & {[f, \bullet]_{B}} & {[s, \bullet]_{B}}\end{array}\right)$ on a basis respecting $W \oplus$ $S \oplus S^{*}$ and $b=w+s+f$. Now, $B$ being quadratic is equivalent to $W$ being quadratic. If $<,>_{W}$ is bi-invariant on $W$, represented by $Z_{W}$, then:

$$
Z_{W \oplus S \oplus S^{*}}=\left(\begin{array}{ccc}
Z_{W} & 0 & 0 \\
0 & 0 & \mathbb{I} \\
0 & \mathbb{I} & 0
\end{array}\right)
$$

is bi-invariant on $B$. In practice, computing a double extension of a quadratic indecomposable non simple $B$ proceeds as follow [14]. We take a minimal ideal $I$ of $B$ and consider $I^{\perp}$ its orthogonal by a bi-invariant $<,>_{B}$. The decomposition $B=W \oplus S \oplus S^{*}$ where $W=I^{\perp} / I, S=B / I^{\perp}$ and $S^{*}=I$ is a double extension of $W$ with $S$ simple (or 1dimensional) and $<,>_{W}=<,>\left._{B}\right|_{W}$ is bi-invariant. Moreover, $I$ and $I^{\perp}$ verify the properties (*): (i) $I$ is abelian, (ii) $I^{\perp}$ is a maximal ideal, (iii) $I \subset I^{\perp}$ and $\left[I, I^{\perp}\right]=0$, (iv) $\operatorname{codim}\left(I^{\perp}\right)=\operatorname{dim}(I)$.

\section{THE ALGORITHM}

For abelian and simple $\mathfrak{g}$, computing the quadratic space $\mathscr{Z}(\mathfrak{g})$ is trivial. An abelian $\mathfrak{g}$ is quadratic and $\mathscr{Z}(\mathfrak{g})=\operatorname{Sym}(n)$ (symmetric matrices). A simple $\mathfrak{g}$ is also quadratic and $\mathscr{Z}(\mathfrak{g})=\mathbb{F} \beta$ is the vector space spanned by its (non-degenerate [15]) Killing form $\beta(x, y)=\operatorname{Tr}(\operatorname{ad}(x) \circ \operatorname{ad}(y))$. Computing $\mathscr{Z}(\mathfrak{g})$ in general is more complicated. Here we present an algorithm that determines if $\mathfrak{g}$ is quadratic and if so, gives a bi-invariant $<,>\mathfrak{g}$.

Elementary bi-invariant $<,>_{\mathfrak{g}}$ of $\mathfrak{g}$. More precisely, the algorithm tries to construct a bi-invariant $<,>_{\mathfrak{g}}$ that exists necessarily on a quadratic $\mathfrak{g}$. We call $<,>_{\mathfrak{g}}$ an elementary bi-invariant $<,>_{\mathfrak{g}}$ of $\mathfrak{g}$. If the algorithm fails to construct it, $\mathfrak{g}$ is not quadratic. The elementary bi-invariant $<,>_{B}$ of a simple $B$ (resp. 1-dim.) is defined to be the Killing 
form (resp. the multiplication). An elementary bi-invariant $<,>_{\mathfrak{g}}$ of a general quadratic $\mathfrak{g}$ - on which we know an auxiliary bi-invariant $<,>-$ is defined using [14]:

Theorem 1 (Medina \& Revoy) $\mathfrak{g}$ is quadratic if and only if its adjoint representation decomposes into indecomposable subrepresentations $B$ that are either simple or 1dimensional (Type (1)), or a double extension $B=W \oplus S \oplus S^{*}$ of $W$ quadratic by $S$ simple of 1-dimensional (Type (2)).

More precisely, we decompose the adjoint representation of $\mathfrak{g}$ into indecomposable $B$ 's. On the $B$ 's of Type (1), we take the elementary bi-invariant $<,>_{B}$ : the Killing form (or the multiplication if $B$ is 1-dim.). On the $B$ 's of Type (2), we build the double extension. We consider a minimal ideal $I$ and, using the auxiliary bi-invariant $<,>$ of $\mathfrak{g}$, we compute $I^{\perp}$. We get the double extension $B=W \oplus S \oplus S^{*}$ with $W=I^{\perp} / I, S=B / I^{\perp}$ and $S^{*}=I$. We construct an elementary bi-invariant $<,>_{W}$ on $W$ recursively. We define an elementary bi-invariant $<,>_{B}$ on the double extension $B=W \oplus S \oplus S^{*}$ to be of the form of Eq.(2). Finally, we define an elementary bi-invariant $<,>_{\mathfrak{g}}$ on the direct sum $\mathfrak{g}=B_{1} \oplus \ldots \oplus B_{N}$ to be of the form of Eq.(1). This construction defines and proves the existence of (at least) one elementary bi-invariant $<,>_{\mathfrak{g}}$ of a quadratic $\mathfrak{g}$.

The algorithm. In the following, we use matrix representations $Z$ of $<$, >, where the basis will be specified. For our purpose, we want to determine if $\mathfrak{g}$ is quadratic and (if so) to exhibit a bi-invariant $Z_{\mathfrak{g}}$. The input is $\mathscr{B}_{\mathfrak{g}}$, a basis of $\mathfrak{g}$ and the Lie bracket $[,]_{\mathfrak{g}}$ on this basis. The idea is a reductio ad absurdum. We assume that $\mathfrak{g}$ is quadratic, although of course, we do not have an auxiliary bi-invariant $Z$. We try to construct an elementary bi-invariant $<,>_{\mathfrak{g}}$ by testing the possible "candidates". If we find one, we return it. If not, we conclude that $\mathfrak{g}$ is not quadratic. More precisely, we decompose the adjoint representation of $\mathfrak{g}$ into indecomposable $B$ 's following Th. (1). On the $B$ 's of Type (1), we take the Killing form. The other $B$ 's are indecomposable and non simple (nor 1-dim.). For each such $B$, we take $I$ a minimal abelian ideal, which is a minimal ideal of $B$. Because we assume that $\mathfrak{g}$ is quadratic, $B$ is quadratic and there exists an ideal $J \subset B$ verifying $(*)$, which is $I^{\perp}$ for an elementary bi-invariant $<_{,}>_{B}$. If not, there is a contradiction and $\mathfrak{g}$ is not quadratic. Otherwise for each $J$, we define $W=J / I$ and $S=B / J$. Note that $S$ is simple (or 1-dim.) since $J$ is maximal. Because $B$ is quadratic, one $J$ is an $I^{\perp}$ and provides a double extension decomposition and an expression of a bi-invariant $Z_{B}$. We examine the two cases : $W=\{0\}$ and $W \neq\{0\}$. For $W=\{0\}$, a bi-invariant $Z_{B}$ on $B=S \oplus I$ has the form : $Z_{B=S \oplus I}=\left(\begin{array}{cc}0 & L_{0} \\ L_{0}^{T} & 0\end{array}\right)$ where $L_{0}$ is an isomorphism of $S$-representations between $S$ and $I$. The basis of $B$ respects the direct sum but there is no reason that the basis for $S$ and $I$ are already "duals". If no such $L_{0}$ exists, there is a contradiction: we conclude that $\mathfrak{g}$ is not quadratic. Otherwise, we perform the change of basis $\left(\mathbb{I}, L_{0}^{-1}\right)$ on $B=S \oplus I$ s.t. $Z_{B=S \oplus I}=\left(\begin{array}{ll}0 & \mathbb{I} \\ \mathbb{I} & 0\end{array}\right)$, which is bi-invariant. For $W \neq\{0\}$, we have $B=W \oplus S \oplus I$. We repeat the reasoning on $W$ (recursivity). If a contradiction is found, $\mathfrak{g}$ is not quadratic. Otherwise, we get a bi-invariant $Z_{W}$ on $W$. Then on $B=W \oplus S \oplus I$, a bi-invariant $Z_{B}$ has the form: $Z_{B=W \oplus S \oplus I}=\left(\begin{array}{ccc}Z_{W} & 0 & 0 \\ 0 & 0 & L_{0} \\ 0 & L_{0}^{T} & 0\end{array}\right)$ 
where $L_{0}$ is an isomorphism of $S$-representations between $S$ and $I$. If no such $L_{0}$ exists, there is a contradiction. Else we have a bi-invariant $Z_{B}$. If we find a $Z_{B}$ for each of the $B$ 's of the decomposition of $\mathfrak{g}$, we find the bi-invariant $Z_{\mathfrak{g}}$ by Eq.(1).

\section{Algorithm 1 (Computation of a bi-invariant pseudo-metric on $\mathfrak{g}$ )}

- Initialization: $B_{\mathfrak{g}}$ basis of $\mathfrak{g}$ and $[,]_{\mathfrak{g}}$ on this basis.

\section{- Recurrence:}

- Case 1: If $B$ is decomposable into $B=B_{1} \oplus B_{2} \oplus \ldots \oplus B_{N}$, call recursively the algorithm on the $B_{i}$ 's; return: $Z_{B}=\operatorname{Diag}\left(Z_{B_{1}}, \ldots, Z_{B_{N}}\right)$

- Case 2: If $B=\{0\}$, return $Z_{B}=(\emptyset)$.

- Case 3: If $B$ is 1-dimensional, return $Z_{B}=(1)$

- Case 4: If B is simple, return $Z_{B}=\operatorname{Tr}(\operatorname{ad}() a d.()$.$) (The Killing form).$

- Case 5: Else take I a minimal abelian ideal of $B$ and $\mathscr{J}=\left\{J_{j}\right\}_{j=1}^{n_{i}}$ the set of maximal ideals verifying properties $(*)$. Until $\mathscr{J}=\emptyset$ : take $J \in \mathscr{J}$; withdraw it from $\mathscr{J}$; compute $S=B / J, W=J / I$; and:

- If $S \nsim$ I: EXIT.

- Else: compute $L_{0}$; perform the change of basis $\left(\mathbb{I}, L_{0}^{-1}\right)$ on $B=S \oplus I$.

* Case $W=\{0\}$ : return: $Z_{B=S \oplus I}=\left(\begin{array}{cc}0 & \mathbb{I} \\ \mathbb{I} & 0\end{array}\right)$

* Case $W \neq\{0\}$ : check double extension structure; call algorithm on $W$;

return: $Z_{B=W \oplus S \oplus I}=\left(\begin{array}{ccc}Z_{W} & 0 & 0 \\ 0 & 0 & \mathbb{I} \\ 0 & \mathbb{I} & 0\end{array}\right)$

- End: if "EXIT": $\mathfrak{g}$ is not quadratic; else expression of a bi-invariant $<,>\mathfrak{g}$.

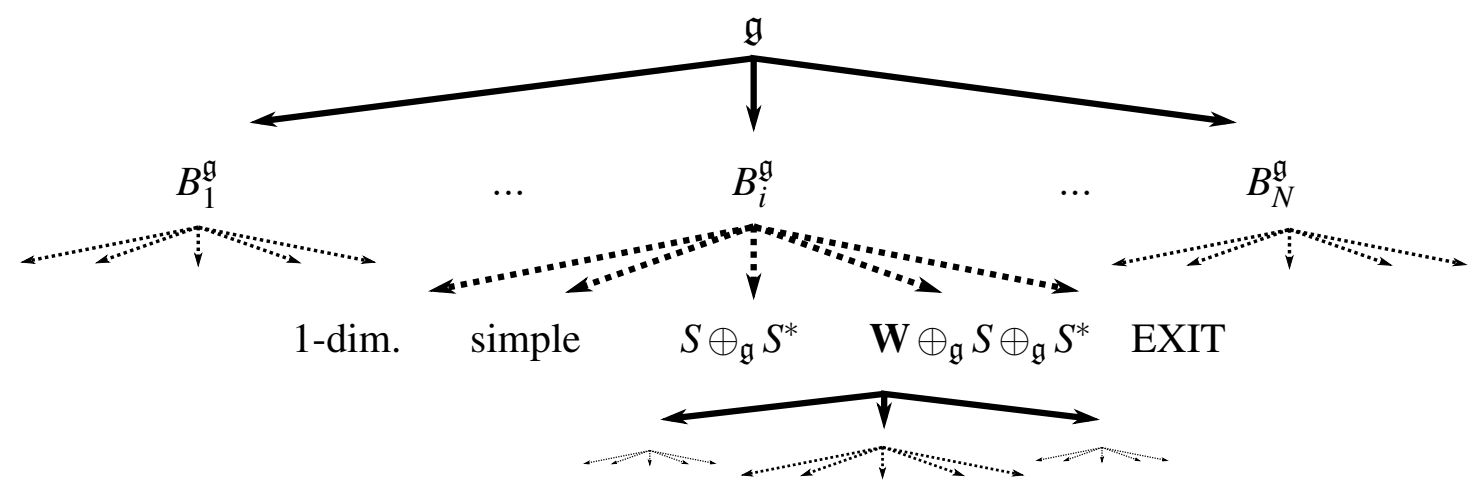

FIGURE 1. Tree structure of the algorithm. A tree level corresponds to a reduction of an adjoint representation: reduction of $\mathfrak{g}$ into $B$ 's for the first level, reductions of the $W$ 's into $B$ 's for the others.

This algorithm has a natural tree structure as shown in Figure 1. The bi-invariant $<,>_{\mathfrak{g}}$ is computed in a postfix manner. To implement the algorithm, auxiliary functions are needed: reduction of $B$, search for a minimal abelian ideal, computation of all maximal ideals, computation of $L_{0}$ etc. They can be found in [16], [17] or written in particular cases. For our purpose of deciding if the pseudo-Riemannian framework 
enables consistent statistics on Lie groups, we perform the algorithm manually on Lie groups which are know to have locally unique bi-invariant mean.

\section{RESULTS ON SELECTED LIE GROUPS}

In this section, we perform Algorithm (1) to find if a bi-invariant pseudo-metric exists on some real Lie groups for which there is a locally unique bi-invariant mean: $S E(n)$, $S T(n), H, U T(n)$. The results show that most of them do not have any bi-invariant $<,>$.

Scalings and translations $S T(n)$. The Lie algebra $\mathfrak{s t}(n)$ is composed of the $(\mu, u) \in$ $\mathbb{R} \oplus \mathbb{R}^{n}$ with Lie Bracket $\left[\left(\mu_{1}, u_{1}\right),\left(\mu_{2}, u_{2}\right)\right]=\left(0, \mu_{2} \cdot u_{1}-\mu_{1} \cdot u_{2}\right)$. We choose the basis $\left(D,\left\{P_{a}\right\}_{a=1 \ldots n}\right)$ defined as: $D=(1,0)$ and $P_{a}=\left(0, e_{a}\right)$ with $\left(e_{a}\right)_{a=1 \ldots n}$ the canonical basis of $\mathbb{R}^{n}$. In this basis: $\left[P_{a}, P_{b}\right]=0,\left[D, P_{a}\right]=P_{a},[D, D]=0$. We start Algorithm 1 and investigate the different cases. We look at Case 1 . We compute all ideals of $\mathfrak{s t}(n)$ and find: $\operatorname{Span}\left(P_{1}\right), \ldots, \operatorname{Span}\left(P_{1}\right)$ and their linear combinations. No ideal contains $D$, thus $\mathfrak{s t}(n)$ is indecomposable, thus we exclude Case 1. We look at Cases 2 and 3. If $n=0$, we are in Case 3 and we have a trivial bi-invariant $<,>$. Otherwise $\operatorname{dim}(\mathfrak{s t}(n))>1$, thus we exclude Cases 2 and 3. We look at Case 4 for $n \neq 0$ : $\operatorname{Span}\left(P_{1}\right)$ for example is an ideal, thus $\mathfrak{s t}(n)$ is not simple, thus we exclude Case 4 . We look at Case 5 for $n \neq 0$. We take $I=\operatorname{Span}\left(P_{1}\right)$. The only maximal ideal verifying $(*)$ is $J=P=\operatorname{Span}\left(\left\{P_{a}\right\}_{a}\right)$. We have $S=\mathfrak{s t}(n) / J=\operatorname{Span}(D)$ and $W=J / I=\operatorname{Span}\left(P_{2}, . . P_{n}\right)$. The $S$-representation on $S$ is the null representation: $[D, D]=0$. The $S$-representation on $I$ is the trivial representation: $\left[D, P_{1}\right]=P_{1}$. Hence, $I \not S$ and we exit the algorithm. We conclude that there is no biinvariant $<,>$ on $\mathfrak{s t}(n)$ for $n \neq 0$.

Heisenberg group $H$ and Scaled Upper Unitriangular Matrices UT $(n)$. Algorithm 1 confirms that $H$ has no bi-invariant $<,>$ [13]. Now $\mathfrak{u t}(n)$ is decomposable into the 1 -dim. Lie algebra generated by $\mathbb{I}$ and the Heisenberg algebra $\mathfrak{h}$. As $\mathfrak{h}$ has no bi-invariant $<,>$, neither does $\mathfrak{u t}(n)$.

Rigid Body Transformations $S E(n)$. The Lie algebra $\mathfrak{s e}(n)$ is composed of the $(A, u) \in \operatorname{Skew}(n) \oplus \mathbb{R}^{n}$ with Lie Bracket $\left[\left(A_{1}, u_{1}\right),\left(A_{2}, u_{2}\right)\right]=\left(A_{1} \cdot A_{2}-A_{2} \cdot A_{1}, A_{1} \cdot u_{2}-\right.$ $\left.A_{2} \cdot u_{1}\right)$. We choose the basis: $\left(\left\{J_{i j}\right\}_{1 \leq i<j \leq n},\left\{P_{a}\right\}_{a=1 \ldots n}\right)$ with $J_{i j}=e_{i} \cdot e_{j}^{T}-e_{j} \cdot e_{i}^{T}$ and $\left\{P_{a}\right\}_{a=1 \ldots n}$ the canonical basis of $\mathbb{R}^{n}$. In this basis: $\left[J_{i j}, J_{k l}\right]=\delta_{i k} . J_{j l}-\delta_{j k} . J_{i l}+\delta_{j l} . J_{i k}-$ $\delta_{i l} . J_{j k},\left[J_{i j}, P_{a}\right]=\delta_{a j} . P_{i}-\delta_{a i} . P_{j},\left[P_{a}, P_{b}\right]=0$, with $\delta$ the Kronecker symbol.

As preliminaries, we show that $P=\operatorname{Span}\left(\left\{P_{a}\right\}_{a}\right)$ is the only proper ideal of $\mathfrak{s e}(n)$. First $P$ is a proper ideal of $\mathfrak{s e}(n)$. Suppose that $\mathfrak{s e}(n)$ has another proper ideal $K$. Then either $K \cap P$ is a proper ideal of $\mathfrak{s e}(n)$ included in $P$ or $K \subset \mathfrak{s o}(n)$ is a proper ideal of $\mathfrak{s e}(n) . P$ does not contain any proper ideal of $\mathfrak{s e}(n)$, because $\mathfrak{s o}(n)$ acts transitively on $P$ with the Lie bracket. We can show that $\mathfrak{s o}(n)$ does not contain any proper ideal of $\mathfrak{s e}(n)$ (considering independently the case $n=4$ ). Thus $P$ is the only proper ideal of $\mathfrak{s e}(n)$.

Now we start Algorithm (1) and look at the different cases. We look at Case 1: $\mathfrak{s e}(n)$ has only one ideal $P$, thus $\mathfrak{s e}(n)$ is indecomposable, thus we exclude Case 1 . We look at Cases 2 and 3. If $n=0$, we are in Case 2 and we have a trivial bi-invariant $<,>$. If $n=1$, we are in Case 3 and the multiplication is a bi-invariant $<,>$. Otherwise $\operatorname{dim}(\mathfrak{s e}(n))>1$, 
thus we exclude Cases 2 and 3. We look at Case 4 for $n \notin\{0,1\}$. $P$ is an ideal, thus $\mathfrak{s e}(n)$ is not simple, thus we exclude Case 4. We look at Case 5 for $n \neq\{0,1\}$. We take $I=P$ and $J=P=I$ because $P$ is the only ideal of $\mathfrak{s e}(n)$. $J$ satisfies condition (iv) of $(*)$ only for $n=3$. We conclude that $\mathfrak{s e}(n)$ does not have any bi-invariant $<,>$ if $n \notin\{0,1,3\}$. For $n=$ 3, we compute $S=\mathfrak{s e}(3) / P \sim \mathfrak{s o}(3)$ and $W=P / P=\{0\}$. To study the $S$-representations, we write the Lie bracket as $\left[J_{m}, J_{n}\right]=\varepsilon_{m n p} . J_{p},\left[J_{m}, P_{a}\right]=\varepsilon_{m a p} . P_{p},\left[P_{a}, P_{b}\right]=0$, where we define $J_{1}=J_{23}, J_{2}=J_{31}$ and $J_{3}=J_{12}$. The $\mathfrak{s o}(3)$-representation on $\mathfrak{s o}(3)$ is the adjoint representation: $\left[J_{m}, J_{n}\right]=\varepsilon_{m n p} . J_{p}$. The $\mathfrak{s o}(3)$-representation on $P$ is given by: $\left[J_{m}, P_{a}\right]=\varepsilon_{\text {map }} . P_{p}$. It is also the adjoint representation. The isomorphism of vector spaces $L_{0}$ that maps each $P_{a}$ on $J_{a}$ is an isomorphism of representations whose matricial form is the identity in our basis. Hence we write $<,>_{\mathfrak{s e}(n)}$ on the decomposition $\mathfrak{s o}(3) \oplus P$ with basis $\left(J_{a}, P_{a}\right)$ and get: $Z_{\mathfrak{s e}(n)}=\left(\begin{array}{cc}0 & \mathbb{I}_{3} \\ \mathbb{I}_{3} & 0\end{array}\right)$. It provides a bi-invariant $<,>_{\mathfrak{s e}(3)}$ on $\mathfrak{s e}(3)$, verified with Eq.(1), and known as the Klein form [18].

\section{REFERENCES}

1. M. Fréchet, L'intégrale abstraite d'une fonction abstraite d'une variable abstraite et son application a la moyenne d'un élément aléatoire de nature quelconque, La Revue Scientifique, (1944).

2. M. Fréchet, Les éléments aléatoires de nature quelconque dans un espace distancié, Annales de l'institut Henri Poincaré, 10, 215-310 (1948).

3. H. Karcher, Riemannian center of mass and mollifier smoothing, Communications on Pure and Applied Mathematics, 30, 509-541 (1977).

4. W. S. Kendall, Probability, Convexity, and Harmonic Maps with Small Image I: Uniqueness and Fine Existence, Proceedings of the London Mathematical Society, s3-61, 371-406 (1990).

5. M. Émery, and G. Mokobodzki, Sur le barycentre d'une probabilité dans une variété, Séminaire de probabilités de Strasbourg, 25, 220-233 (1991).

6. J. M. Corcuera, and W. S. Kendall, Riemannian Barycentres and Geodesic Convexity, Math. Proc. Cambridge Philos. Soc, 127 (1998).

7. L. Huiling, Estimation of Riemannian Barycentres, LMS Journal of Comput. and Math., 7, (2004).

8. L. Yang, Riemannian median and its estimation, LMS Journal of Comput. and Math., 13, (2010).

9. B. Afsari, Riemannian $L^{p}$ center of mass: existence, uniqueness, and convexity., Proc. Am. Math. Soc., 139, (2011).

10. D. Thompson, and J. Bonner, On Growth and Form, Canto (Cambridge University Press), Cambridge University Press, 1992.

11. X. Pennec, and V. Arsigny, Exponential Barycenters of the Canonical Cartan Connection and Invariant Means on Lie Groups, Matrix Information Geometry, Springer, 2012, pp. 123-168.

12. S. Sternberg, Lectures on differential geometry (1964), autre tirage : 1965.

13. A. Medina, Groupes de lie munis de pseudo-métriques de riemann bi-invariantes, Sémin. géométrie différentielle 1981-1982, Montepellier (1982).

14. A. Medina, and P. Revoy, Algèbres de Lie et produit scalaire invariant, Annales scientifiques de l'E.N.S., 18, (1985).

15. E. Cartan, Sur la structure des groupes de transformations finis et continus, Vuibert, (1933).

16. D. Rand, P. Winternitz, and H. Zassenhaus, On the identification of a Lie algebra given by its structure constants. I. Direct decompositions, levi decompositions, and nilradicals, Linear Algebra and its Applications, 109, (1988).

17. M. Ceballos, J. Núñez, and A. F. Tenorio, Algorithmic Method to Obtain Abelian Subalgebras and Ideals in Lie Algebras, Int. J. Comput. Math., 89, (2012).

18. A. Karger, and J. Novák, Space kinematics and Lie groups, Gordon and Breach Science Publishers, (1985) 